\title{
Role of Ultrafast MR Imaging in Stroke Patients
}

\author{
Manne Sindhura ${ }^{1}$ Rajeswaran Rangasami ${ }^{1} \quad$ Anupama Chandrasekharan ${ }^{1}$ \\ ${ }^{1}$ Department of Radiology and Imaging Sciences, Sri Ramachandra \\ Institute of Higher Education and Research (DU), Porur, Chennai, \\ India

\begin{abstract}
Address for correspondence Rajeswaran Rangasami, MD, DNB, PhD, Department of Radiology and Imaging Sciences, Sri Ramachandra Institute of Higher Education and Research (DU), Porur, Chennai 600 116, India (e-mail: rrajeswaran2000@gmail.com).
\end{abstract}

J Neurosci Rural Pract:2020;11:436-441

\begin{abstract}
Objectives The aim of the study is to assess the role of ultrafast (UF) magnetic resonance (MR) sequences in stroke imaging.

Material and Methods We prospectively studied 85 patients having clinical suspicion of stroke referred for MR imaging (MRI) during August 2016 to July 2018. These patients were subjected to both conventional and UF MRI sequences. The patients were divided into six categories based on the pathologies encountered. Further subclassification was done based on the size of the lesions as $\leq 10 \mathrm{~mm}$ and $>10 \mathrm{~mm}$ as seen separately in both UF and conventional MR sequences. The number and visibility of these lesions on conventional and UF MRI were compared. The image quality of all the subjects was also compared based on a scale categorized into excellent, satisfactory, and poor. The findings on conventional and UF imaging sequences were correlated with the final clinical diagnosis arrived at the time of discharge.

Results In our study comprising 85 patients, 57 showed pathologies. The patients showing pathologies were assigned into the six categories as acute infarct (34 cases), acute hemorrhagic infarct (six cases), chronic infarct (17 cases), chronic hemorrhagic infarct (four cases), subacute infarct (three cases), and chronic hemorrhage (one case). The number of lesions seen on conventional and UF sequences were the same although there was a slight decrease in the size of the lesions on UF sequences as compared with conventional counterparts. The image quality using UF sequences

\section{Keywords}

- ultrafast MR imaging

- cerebral infarct

- cerebral hemorrhage

- CVA

- CVI was better in motion prone patients while conventional imaging showed better image quality in cooperative patients.

Conclusion In motion prone patients, UF sequences are a suitable alternative for conventional sequences as they help in arriving at the diagnosis in lesser time, with reasonably good image quality, and without motion artifacts. In cooperative stroke patients, it is better to use conventional MR sequences as the image quality is better.
\end{abstract}

\section{Introduction}

Ultrafast (UF) magnetic resonance (MR) imaging (MRI) sequencing is a technique that enables shortening of MR examination time, decreases the need for sedation, assesses neurological morphology as well as function, and increases patient throughput. ${ }^{1-3}$ The duration of each sequence is 20 to 40 seconds. UF MR sequences are useful in brain pathologies, abdominal studies, pediatric brain, and fetal stroke. ${ }^{4-13}$ Sometimes stroke patients present with restlessness, movement, and disorientation. In these circumstances, UF sequences are useful to arrive at a diagnosis with half $\mathrm{K}$-space sampling, thus serving as an alternative tool. ${ }^{14}$

\section{Materials and Methods}

MRI was performed with a 1.5-T superconducting system (GE Signa HDX 1.5T) with an 8 Channel Neuro Vascular Array/16 
Channel Head Neck Spine Coil. A prospective observational study was conducted at our institution on 85 patients between July 2016 and August 2018 with features of stroke on clinical examination. This prospective study was approved by the Institution Ethics Committee. The patients underwent both conventional and UF MRI protocols in a single study as a part of the protocol. The conventional MR sequences protocol included T1 axial or sagittal using T1 fluid-attenuated inversion recovery (FLAIR) sequence, T2 axial using fast spin echo sequence (FSE), gradient echo imaging (GRE) axial with total scan time of 4.08 minutes Sequences namely T2 FLAIR, diffusion-weighted imaging, and three-dimensional time of flight MR angiogram were also done. Additionally, axial sections of T2 half-Fourier-acquisition single shot turbo spin echo (HASTE), T1 fast spoiled gradient echo (FSPGR), and echo-planar imaging-gradient echo imaging (EPI-GRE) were performed as UF sequence imaging with total scan time of 2.0 minutes The parameters of the sequences used are shown in - Table 1.

Out of these 85 patients, 28 patients did not show any abnormality on both conventional and UF MRI. They were normal on clinical follow-up. However, 57 patients showed pathologies and were divided into six categories as follows:

1. Acute infarct.

2. Acute hemorrhagic infarct.

3. Chronic infarct.

4. Chronic hemorrhagic infarct.

5. Chronic hemorrhage.

6. Subacute infarct.

Each category of the pathologies was further subclassified based on their size as $<10 \mathrm{~mm}$ and $>10 \mathrm{~mm}$ as seen separately in both UF and conventional MR sequences. The number and visibility of these lesions were compared. For the management of patients, both sets of images were used concurrently in making a diagnosis. For study purposes, the images obtained from conventional sequences and UF sequences were read independently at two different points of time by two senior radiologists with more than 10-year experience. When there was discrepancy, the findings were finalized based on consensus. The image quality of the 85 patients were also assessed and categorized into excellent, satisfactory, and poor based on the image degradation by the artifacts. The findings on conventional and UF imaging sequences were correlated with the final clinical diagnosis arrived at the time of discharge and statistical analysis was performed to ascertain if there was any difference in the visibility of pathologies between the two scanning methods.

\section{Statistical Analysis}

All statistical analysis was performed using Statistical Package for Social Science (SPSS, version 17) for Microsoft windows. The data were not normally distributed and therefore parametric and/or nonparametric tests were performed. The data were expressed as mean and standard deviation. Independent sample student $t$-tests and/or Mann Whitney $\mathrm{U}$ tests were used to compare continuous variables between the two groups. Chi-square test was used for association between the two attributes.

\section{Results}

Of the 85 patients in our study, 36 were females and 49 were males with a mean age of 58 years 6 months. These 85 patients were categorized as cooperative and motion prone based on their cooperativeness during the scan. Out of the 85 patients, 57 patients showed a total of 197 pathologies and 28 patients showed a normal scan. The patients showing pathologies were classified into the six categories as acute infarct (34), acute hemorrhagic infarct (six), chronic infarct (17), chronic hemorrhagic infarct (CHI; four), subacute infarct (three), and chronic hemorrhage (one) (-Table 2). Some patients showed more than one type of pathology. These 57 patients were also evaluated based on the size of the lesions into two subcategories as lesions with size $\leq 10 \mathrm{~mm}$ and lesions $>10 \mathrm{~mm}$ in size.

\section{Cooperative Patients, Pathologies $\leq 10$-mm Size}

In cooperative patients showing acute infarcts $\leq 10 \mathrm{~mm}$ in size, more lesions were seen on UF MRI (23 lesions) as

Table 1 Showing parameters used in the various MR sequences

\begin{tabular}{|c|c|c|c|c|c|c|c|c|}
\hline $\begin{array}{l}\text { Pulse } \\
\text { sequence }\end{array}$ & $\begin{array}{l}\text { TR } \\
\text { (ms) }\end{array}$ & $\begin{array}{l}\text { TE } \\
\text { (ms) }\end{array}$ & Matrix & NEX & FOV & $\begin{array}{l}\text { Slice } \\
\text { thickness } \\
(\mathrm{mm})\end{array}$ & $\begin{array}{l}\text { Spacing } \\
(\mathrm{mm})\end{array}$ & Scan time \\
\hline T2 axial & 3,400 & 106 & $320 \times 256$ & 1 & 26 & 5 & 0.5 & 1.22 \\
\hline DWI & 6,400 & 105 & $128 \times 192$ & - & 26 & 5.0 & 0.5 & 0.58 \\
\hline T2-FLAIR & 9,000 & 120 & $256 \times 160$ & 1 & 22 & 5.0 & 0.5 & 2.17 \\
\hline T1-sagittal & 2,525 & 24 & $256 \times 160$ & 1 & 25 & 5.0 & 0.5 & 1.08 \\
\hline GRE axial & 800 & 30 & $256 \times 160$ & 1 & 24 & 5.0 & 1.5 & 1.38 \\
\hline 3D-TOF & 20 & 3.2 & $256 \times 192$ & - & 24 & 1.4 & - & 2.07 \\
\hline FSPGR & 200 & 1.6 & $392 \times 192$ & 2 & 26 & 5 & 0.5 & 50 \\
\hline SSFSE & 200 & 90 & $256 \times 224$ & 1 & 26 & 5 & 0.5 & 40 \\
\hline GRE-EPI & 1,125 & 10 & $128 \times 192$ & 3 & 26 & 5 & 0.5 & 30 \\
\hline
\end{tabular}

Abbreviations: DWI, diffusion-weighted imaging; FLAIR, fluid-attenuated Inversion recovery; FOV, field of view; FSPGR, fast spoiled gradient echo; GRE-EPI, gradient echo imaging-echo-planar imaging; MR, magnetic resonance; NEX, number of excitations; SSFSE, single shot fast spin echo; TE, echo time; TOF, time of flight; TR, repetition time. 
Table 2 Showing the different pathologies encountered

\begin{tabular}{|l|l|l|}
\hline S. no. & Pathology & No. of cases \\
\hline 1 & Acute infarct & 34 \\
\hline 2 & Acute hemorrhagic infarct & 6 \\
\hline 3 & Chronic infarct & 17 \\
\hline 4 & Chronic hemorrhagic infarct & 4 \\
\hline 5 & Subacute infarct & 3 \\
\hline 6 & Chronic hemorrhage & 1 \\
\hline
\end{tabular}

compared with conventional MRI (18 lesions). The apparent increase in the number was because of $>10-\mathrm{mm}$ sized infarcts on conventional imaging appeared as $<10-\mathrm{mm}$ sized infarcts on UF imaging ( - Fig. 1A).

In cooperative patients with chronic nonhemorrhagic infarcts (CIs) and CHIs with lesions $\leq 10 \mathrm{~mm}$ in size, UF imaging showed all the lesions (19 lesions in $\mathrm{Cl}$ and seven lesions in $\mathrm{CHI}$ ) as seen in conventional imaging with no

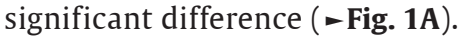
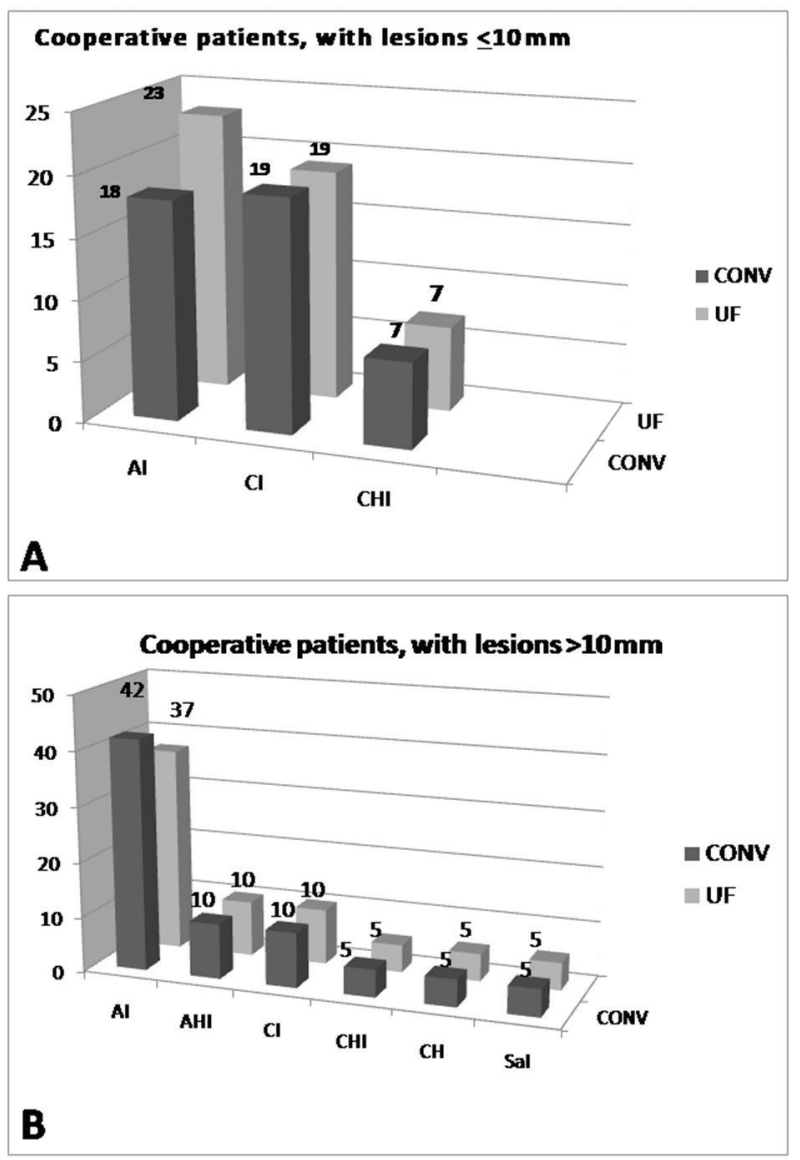

Fig. 1 (A) Visibility of acute, chronic, and chronic hemorrhagic infarcts in both conventional and ultrafast sequences in cooperative patients, when the sizes of the lesions were $<10 \mathrm{~mm}$. (B) Visibility of various pathologies in both conventional and ultrafast sequences in cooperative patients, when the sizes of the lesions were $>10 \mathrm{~mm}$. AHI, acute hemorrhagic infarct; $\mathrm{Al}$, acute infarct; $\mathrm{CH}$, chronic hemorrhage; $\mathrm{CHI}$, chronic hemorrhagic infarct; $\mathrm{Cl}$, chronic infarct; Sal, subacute infarct.

\section{Cooperative Patients, Pathologies $>\mathbf{1 0}-\mathbf{m m}$ Size}

In the patients showing acute infarcts $>10 \mathrm{~mm}$ in size, more number of lesions were seen on conventional MRI in cooperative ( 42 lesions) patients as compared with UF MRI (37 lesions) ( - Fig. 1B). In cooperative patients showing acute hemorrhagic infarcts (ten lesions), chronic infarcts (ten lesions), CHI (five lesions), chronic hemorrhage (five lesions), and subacute infarcts (five lesions), UF imaging showed all the lesions as seen in conventional imaging ( - Fig. 1B).

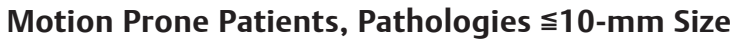

In motion prone patients showing acute infarcts $\leq 10 \mathrm{~mm}$ in size, more lesions were seen on UF imaging (14 lesions) sequences as compared with conventional imaging (ten

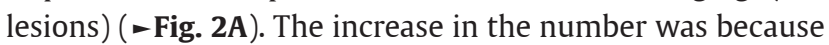
of $>10-\mathrm{mm}$ sized infarcts appearing as $<10-\mathrm{mm}$ sized infarcts on UF imaging. In patients having $\mathrm{CI}$ with lesions $<10 \mathrm{~mm}$ in size, UF imaging showed all the lesions (two lesions), as seen in conventional MRI with no significant difference. None of the motion prone patients in this study showed $\mathrm{CHI}$ with lesions measuring $\leq 10 \mathrm{~mm}$.
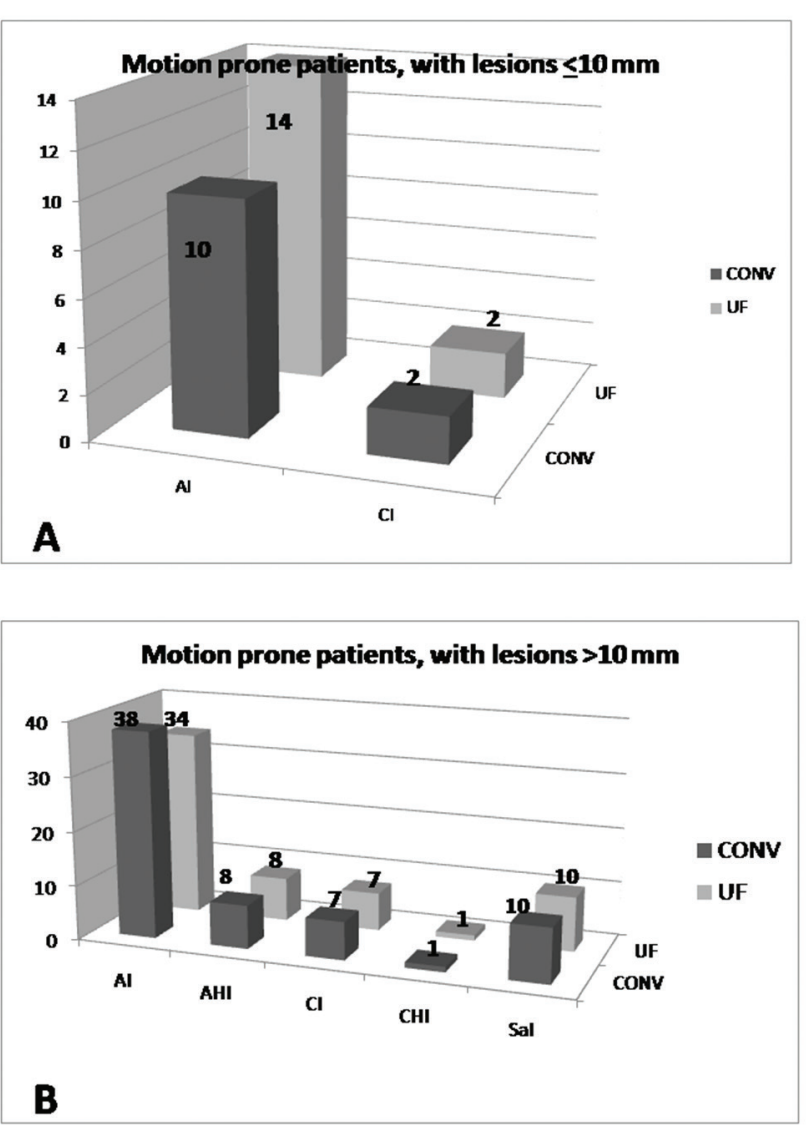

Fig. 2 (A) Visibility of acute and chronic infarcts in both conventional and ultrafast sequences in motion prone patients, when the sizes of the lesions were $\leq 10 \mathrm{~mm}$. (B) Visibility of various pathologies in both conventional and ultrafast sequences in motion prone patients, when the sizes of the lesions were $>10 \mathrm{~mm}$. AHI, acute hemorrhagic infarct; $\mathrm{Al}$, acute infarct; $\mathrm{CH}$, chronic hemorrhage; $\mathrm{CHI}$, chronic hemorrhagic infarct; $\mathrm{Cl}$, chronic infarct; Sal, subacute infarct. 


\section{Motion Prone Patients, Pathologies $>\mathbf{1 0}-\mathrm{mm}$ Size}

In motion prone patients showing acute infarcts $>10 \mathrm{~mm}$ in size, more number of lesions were seen on conventional MRI (38 lesions), as compared with UF MRI (34 lesions) (-Fig. 2B). In patients showing acute hemorrhagic infarcts (eight lesions), chronic infarct (seven lesions), $\mathrm{CHI}$ (one lesion) and subacute infarcts (ten lesions), UF MRI showed all the lesions as seen in conventional MRI ( - Figs. 2B, 3, and 4). None of the motion prone patients showed chronic hemorrhage in this category.

\section{Comparison of Image Quality in Cooperative Patients}

In cooperative patients, the number of patients showing excellent quality images was high with conventional imaging (24 patients) compared with the UF imaging (eight patients). With UF imaging, more images were in the satisfactory category (42 patients) as compared with the conventional imaging (25 patients) (-Fig. 5).

\section{Comparison of Image Quality in Motion Prone Patients} In motion prone patients, the number of patients showing poor quality images was high with conventional imaging (19 patients). With UF imaging, more images were in the satisfactory category (28 patients) (-Fig. 6).

On statistical analysis of the 85 patients who were subjected to conventional and UF imaging, significant number of the patients showed satisfactory imaging quality with $p$-value less than 0.05 (significant) on UF imaging, as compared with conventional imaging.

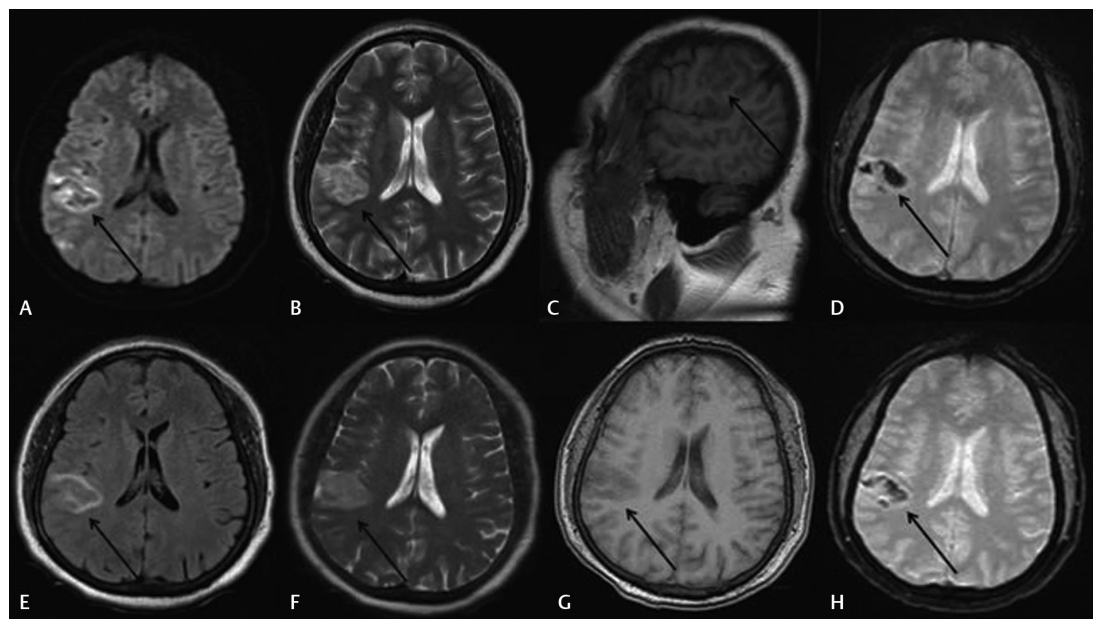

Fig. 3 A 38-year-old (motion prone) male with complaints of left-sided weakness. (A) Axial DWI, (B) axial T2WI, sagittal T1WI, (C) GRE, (D) and axial T2 FLAIR (E) images show acute hemorrhagic infarct (arrow) in the right frontoparietal lobe. Similar findings are seen in T2 HASTE (F), T1 FSPGR (G), and EPI-GRE images (H). No motion artifact is seen in ultrafast sequences (satisfactory quality) whereas conventional images show motion artifact in some of the images (poor quality). DWI, diffusion-weighted imaging; EPI, echo planar imaging; FLAIR, fluid-attenuated Inversion recovery; FSPGR, fast spoiled gradient echo; GRE, gradient echo imaging; HASTE, half-Fourier-acquisition single shot turbo spin echo; T1WI, T1-weighted image; T2WI, T2-weighted image.

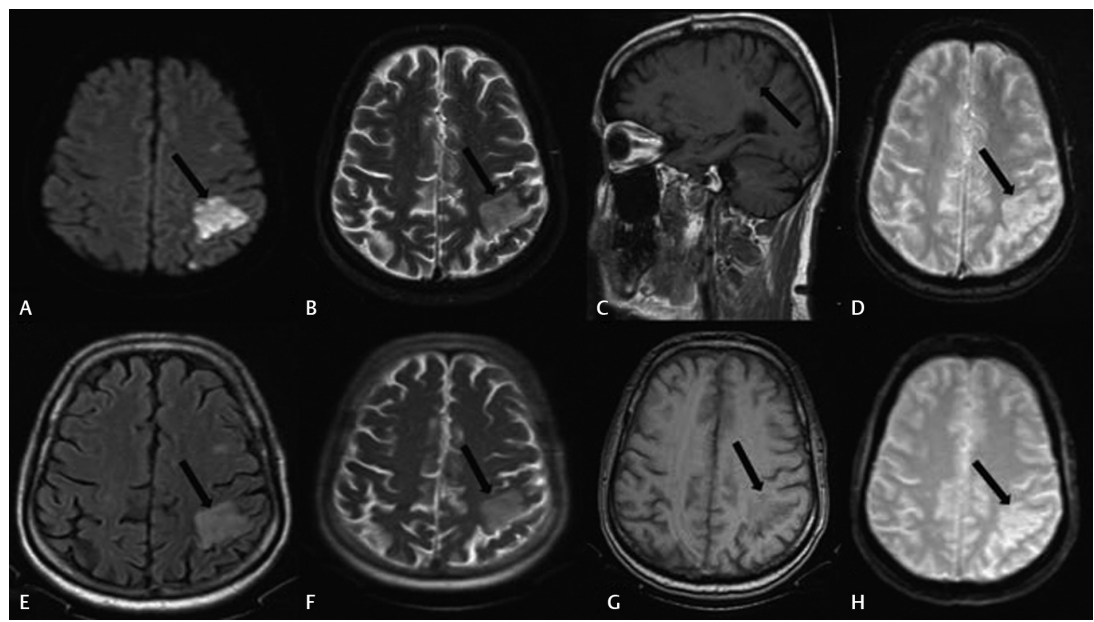

Fig. 4 A 56-year-old male (motion prone) presenting with complaints of right-sided weakness with slurring of speech and giddiness $\times 1$ week. Axial DWI (A), axial T2WI (B), sagittal T1WI (C), GRE (D) and axial T2 FLAIR (E) images showing multiple subacute infarcts (bold arrow) in the left parietal lobe. Similar findings are seen in T2 HASTE (F), T1 FSPGR (G), and EPI-GRE (H). Motion artifact is seen in ultrafast images (satisfactory quality) and conventional images (satisfactory quality). DWI, diffusion-weighted imaging; EPI, echo planar imaging; FLAIR, fluid-attenuated Inversion recovery; FSPGR, fast spoiled gradient echo; GRE, gradient echo imaging; HASTE, half-Fourier-acquisition single shot turbo spin echo; T1WI, T1-weighted image; T2WI, T2-weighted image. 


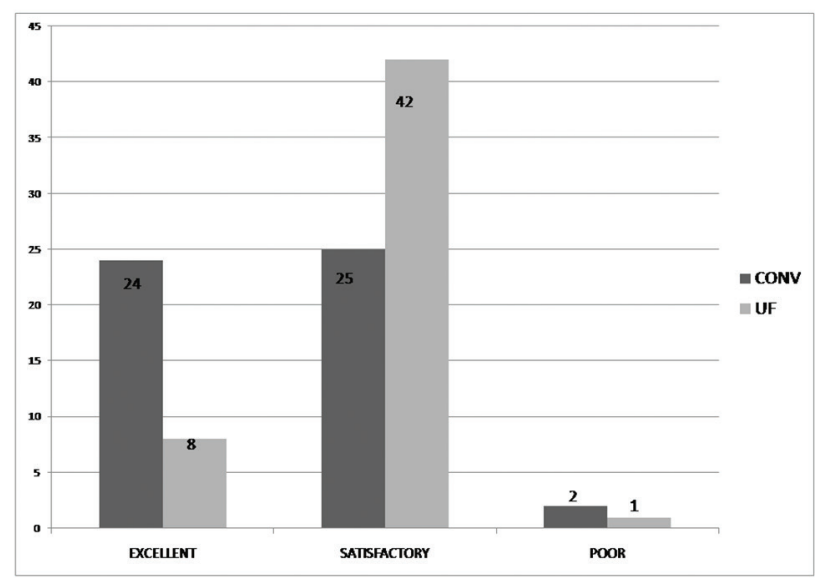

Fig. 5 Comparison of quality of images in conventional and UF sequences in cooperative patients $(n=51)$. UF, ultrafast.

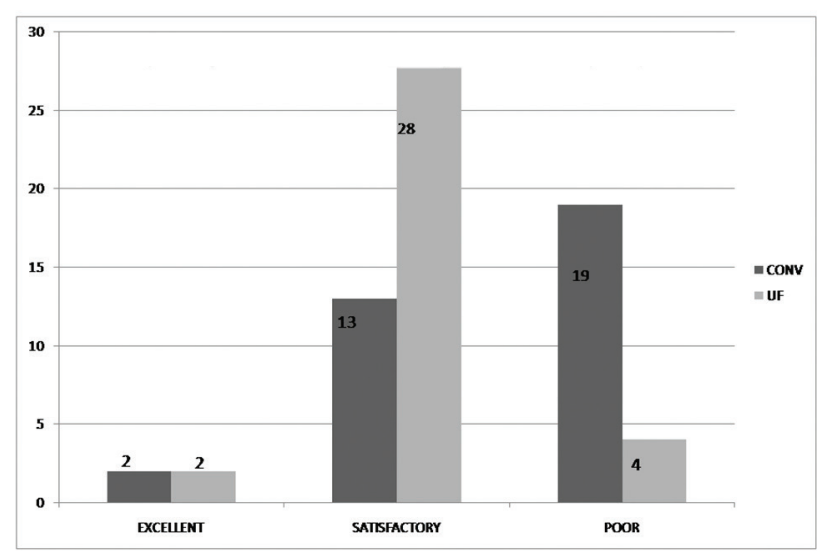

Fig. 6 Comparison of quality of images in conventional and UF sequences in motion prone patients $(n=34)$. UF, ultrafast.

\section{Discussion}

We evaluated 85 people who were referred with features of stroke on clinical examination. All the patients underwent MR brain scans using both conventional and UF sequences in a single clinical study. Out of the total 85 patients studied, 28 patients did not have any pathology while 57 patients showed pathologies. The pathologies were classified into six groups (-Table 2 ) similar to a study done by Prakkamakul et al..$^{5}$ These authors had studied 59 patients using conventional and UF imaging and categorized the pathologies into intracranial mass-like lesion, intracranial hemorrhage, ischemia, and other lesions with diffusion restriction, white matter hyperintensities, subarachnoid FLAIR hyperintensities, and hydrocephalus. ${ }^{5}$

In our study, the pathologies were further subdivided based on their size as $\leq 10 \mathrm{~mm}$ and $>10 \mathrm{~mm}$. These lesions thus seen separately in UF and conventional sets of MR sequences were compared. Patel et $\mathrm{al}^{2}$ studied 34 critically ill patients over the age of 50 years with infarcts or suspected demyelinating disease and also assessed the presence of hemosiderin deposits and motion artifacts using conventional and UF imaging. They visually counted the number of lesions that were $5 \mathrm{~mm}$ or larger and those that were smaller than $5 \mathrm{~mm}$. In our study, we used $10 \mathrm{~mm}$ as the cut off size as there were very few cases whose sizes were less than $5 \mathrm{~mm}$.

\section{Cooperative Patients}

There were 60 acute infarcts on conventional MRI (-Fig. 1A, 1B). All the 60 lesions were also seen on UF sequences although there was a slight reduction in the size of the lesions. All the lesions seen under acute hemorrhagic infarcts (ten lesions), chronic infarcts (29 lesions), CHIs (12 lesions), chronic hemorrhages (five lesions), and subacute infarcts (five lesions) were equally seen on UF sequences as they were seen on conventional sequences ( - Fig. 1A, 1B).

\section{Motion Prone Patients}

There were 48 acute infarcts on conventional MR imaging. All the 48 lesions were also seen on UF sequences, although there was slight reduction in the size of the lesions. Other categories like acute hemorrhagic infarcts (eight lesions), chronic infarcts (nine lesions), $\mathrm{CHI}$ (one lesion), and subacute infarcts (ten lesions) were equally detected on both UF and conventional sequences ( - Fig. 2A, 2B). We did not encounter chronic hemorrhage in any of the motion prone patients.

\section{Quality of the Images}

The image quality of the 85 patients was also assessed and categorized into excellent (excellent quality images without motion artifacts), satisfactory (good images with minor artifacts), and poor (poor quality images with substantial motion artifact) quality based on image degradation by the artifacts.

On statistical analysis of the 85 patients who were subjected to conventional and UF imaging, significant number of the patients showed satisfactory imaging quality with $p$-value less than 0.05 (significant) on UF imaging, as compared with the number of patients exhibiting excellent or poor imaging quality on conventional imaging.

Prakkamakul et $\mathrm{al}^{5}$ in their study categorized the quality of the images based on the image degradation occurring as a result of motion artifact using a five-point scale and concluded that in motion prone patients UF imaging gave better image quality with reduced scan time. Our study findings correlate with this study. We found that there was a mild lag in resolution of the images on UF sequences, but the scan time substantially got reduced.

In the study done by Patel et $\mathrm{al}^{2}$ a subjective grading system was used to assess diagnostic usefulness, degree of periventricular change, lesion conspicuity, and gray-white matter differentiation. These characteristics were graded from 0 to 3, with 0 being the worst and 3 being the best. They concluded that conventional T2-weighted images were better as compared with HASTE images in depicting lesions. In the study by Patel et $\mathrm{al}^{2}$ all the patients were cooperative. The findings in our study correlate with this study as the image quality in conventional imaging was better than UF imaging in cooperative patients.

Singh et $\mathrm{a}^{10}$ in a study on 125 children had compared UF sequences-single shot fast spin echo and EPI T1 sequence with the standard MR sequences and found that the 
sensitivity and specificity for UF imaging were 78 and $98 \%$, respectively. However, they have also mentioned that subtle abnormalities may be missed on UF imaging. Tekes et $\mathrm{al}^{13}$ retrospectively studied MRI scans of 101 children and found that UF imaging is useful for conditions like ventriculomegaly, macrocephaly, and cysts.

UF imaging is being used in some centers as an alternative method for imaging pediatric brain to avoid sedation and radiation from $\mathrm{CT}^{9-13,15}$ In the present study, we not only analyzed the usefulness of UF imaging in cooperative patients but also in motion prone patients. UF imaging can be done rapidly and avoids the need for sedation in motion prone stroke patients. By substituting T2 FSE, T1 FLAIR, and GRE by UF sequences HASTE, FSPGR, and EPI-GRE in motion prone patients, we obtained satisfactory image quality and sufficient information for planning management. These sequences provide information about ischemic as well as hemorrhagic lesions in a shorter time.

\section{Conclusion}

In motion prone patients with suspected stroke, UF MR sequences is a suitable alternative to conventional MR sequences as they help in arriving at the diagnosis in lesser time with reasonably good image quality and relative elimination of motion artifacts. In cooperative stroke patients, it is better to use conventional MR sequences as the image quality is better and the lesions are better appreciated.

\section{Authors' Contributions}

M.S., R.R., and A.C. contributed toward the conceptualization and study design of the manuscript.

\section{Funding}

None.

\section{Conflict of Interest}

None declared.

\section{References}

1 Schuierer G, Reimer P, Allkemper T, Peters PE. Fast and ultra-fast MRI of the brain [in German]. Radiologe 1995;35(12):894-901
2 Patel MR, Klufas RA, Alberico RA, Edelman RR. Half-Fourier acquisition single-shot turbo spin-echo (HASTE) MR: comparison with fast spin-echo MR in diseases of the brain. AJNR Am J Neuroradiol 1997;18(9):1635-1640

3 Ba-Ssalamaha A, Schick S, Heimberger K, et al. Ultrafast magnetic resonance imaging of the brain. Magn Reson Imaging 2000;18(3):237-243

4 U-King-Im JM, Trivedi RA, Graves MJ, et al. Utility of an ultrafast magnetic resonance imaging protocol in recent and semi-recent strokes. J Neurol Neurosurg Psychiatry 2005;76:1002-1005

5 Prakkamakul S, Witzel T, Huang S, et al. Ultrafast brain MRI: clinical deployment and comparison to conventional brain MRI at 3T. J Neuroimaging 2016;26(5):503-510

6 Yamashita S, Masui T, Katayama M, et al. T2-weighted MRI of rectosigmoid carcinoma: comparison of respiratory-triggered fast spin-echo, breathhold fast-recovery fast spin-echo, and breathhold single-shot fast spin-echo sequences. J Magn Reson Imaging 2007;25(3):511-516

7 Baburaj R, Rangasami R, Chandrasekharan A, Suresh I, Suresh S, Seshadri S. Utility of various ultrafast magnetic resonance sequences in the detection of fetal intracranial hemorrhage. Ann Indian Acad Neurol 2018;21(4):275-279

8 Ringelstein EB. Ultrafast magnetic resonance imaging protocols in stroke. J Neurol Neurosurg Psychiatry 2005;76(7):905

9 Ryan ME, Jaju A, Ciolino JD, Alden T. Rapid MRI evaluation of acute intracranial hemorrhage in pediatric head trauma. Neuroradiology 2016;58(8):793-799

10 Singh RK, Smith JT, Wilkinson ID, Griffiths PD. Ultrafast MR imaging in pediatric neuroradiology. Acta Radiol 2003;44(5):550-557

11 Missios S, Quebada PB, Forero JA, et al. Quick-brain magnetic resonance imaging for nonhydrocephalus indications. J Neurosurg Pediatr 2008;2(6):438-444

12 Ashley WW Jr, McKinstry RC, Leonard JR, Smyth MD, Lee BC Park TS. Use of rapid-sequence magnetic resonance imaging for evaluation of hydrocephalus in children. J Neurosurg 2005;103(suppl 3) :124-130

13 Tekes A, Senglaub SS, Ahn ES, Huisman TAGM, Jackson EM. Ultrafast brain MRI can be used for indications beyond shunted hydrocephalus in pediatric patients. AJNR Am J Neuroradiol 2018;39(8):1515-1518

14 Sugahara T, Korogi Y, Hirai T, et al. Comparison of HASTE and segmented-HASTE sequences with a T2-weighted fast spinecho sequence in the screening evaluation of the brain. AJR Am J Roentgenol 1997;169(5):1401-1410

15 Rozovsky K, Ventureyra EC, Miller E. Fast-brain MRI in children is quick, without sedation, and radiation-free, but beware of limitations. J Clin Neurosci 2013;20(3):400-405 